\title{
Hadronic resonance production with ALICE at the LHC
}

\author{
Sergey Kiselev for the ALICE collaboration ${ }^{1, *}$ \\ ${ }^{1}$ Institute for Theoretical and Experimental Physics, 117218 Moscow, Russia
}

\begin{abstract}
.
We present recent results on short-lived hadronic resonances obtained in the ALICE experiment at LHC energies, including results from the Xe-Xe run. The ALICE results on transverse momentum spectra, yields, their ratio to long-lived particles, and nuclear modification factors will be discussed. The results will be compared with model predictions and measurements at lower energies.
\end{abstract}

Hadronic resonances are an important probe for the properties of the medium formed in heavy-ion collisions. In heavy-ion collisions, since the lifetimes of short-lived resonances are comparable with the lifetime of the late hadronic phase, regeneration and rescattering effects become important and resonance ratios to longer lived particles can be used to estimate the time interval between the chemical and kinetic freeze-out [1]. The measurements in pp and p$\mathrm{Pb}$ collisions constitute a reference for nuclear collisions and provide information for tuning event generators inspired by Quantum Chromodynamics.

Recent results on short-lived mesonic $\rho(770)^{0}, \mathrm{~K}^{*}(892)^{0}, \phi(1020)$ as well as baryonic $\Lambda(1520)$ and $\Xi(1530)^{0}$ resonances (hereafter $\rho^{0}, \mathrm{~K}^{* 0}, \phi, \Lambda^{*}, \Xi^{* 0}$ ) obtained in the ALICE experiment are presented. The $\rho^{0}$ has been measured in pp and $\mathrm{Pb}-\mathrm{Pb}$ collisions at $\sqrt{s_{\mathrm{NN}}}=$ $2.76 \mathrm{TeV}$ and results were published recently in [2]. The $\mathrm{K}^{* 0}$ and $\phi$ have been measured in pp collisions at $\sqrt{s}=13 \mathrm{TeV}$, in Xe-Xe collisions at $\sqrt{s_{\mathrm{NN}}}=5.44 \mathrm{TeV}$ and in $\mathrm{Pb}-\mathrm{Pb}$ collisions at $\sqrt{s_{\mathrm{NN}}}=5.02 \mathrm{TeV}$ (results for the $\mathrm{K}^{* 0}$ and $\phi$ in pp at $\sqrt{s}=7 \mathrm{TeV}, \mathrm{p}-\mathrm{Pb}$ at $\sqrt{s_{\mathrm{NN}}}=5.02 \mathrm{TeV}$ and $\mathrm{Pb}-\mathrm{Pb}$ at $\sqrt{s_{\mathrm{NN}}}=2.76 \mathrm{TeV}$ were published in [3], [4] and [5, 6], respectively). The $\Lambda^{*}$ has been measured in $\mathrm{Pb}-\mathrm{Pb}$ collisions at $\sqrt{s_{\mathrm{NN}}}=2.76 \mathrm{TeV}$ and results were published recently in [7]. The $\Xi^{* 0}$ has been measured in $\mathrm{Pb}-\mathrm{Pb}$ collisions at $\sqrt{s_{\mathrm{NN}}}=2.76 \mathrm{TeV}$ (results for the $\Sigma^{* \pm}$ and $\Xi^{* 0}$ in pp at $\sqrt{s}=7 \mathrm{TeV}$ and $\mathrm{p}-\mathrm{Pb}$ at $\sqrt{s_{\mathrm{NN}}}=5.02 \mathrm{TeV}$ were published in [8] and [9], respectively).

The resonances are reconstructed in their hadronic decay channels and have very different lifetimes as shown in Tab. 1.

Table 1. Reconstructed decay mode, branching ratio and lifetime values for hadronic resonances

\begin{tabular}{cccccc}
\hline & $\rho^{0}$ & $\mathrm{~K}^{* 0}$ & $\phi$ & $\Lambda^{*}$ & $\Xi^{* 0}$ \\
\hline decay channel (B.R.) & $\pi \pi(1.00)$ & $\mathrm{K} \pi(0.67)$ & $\mathrm{KK}(0.49)$ & $p \mathrm{~K}(0.22)$ & $\Xi \pi(0.67)$ \\
lifetime (fm/c) & 1.3 & 4.2 & 46.2 & 12.6 & 21.7 \\
\hline
\end{tabular}

Fig. 1 presents the transverse momentum spectra for $\mathrm{K}^{* 0}$ and $\phi$ in $\mathrm{Xe}-\mathrm{Xe}$ collisions at $\sqrt{s_{N N}}=5.44 \mathrm{TeV}$.

\footnotetext{
*e-mail: Sergey.Kiselev@cern.ch
} 

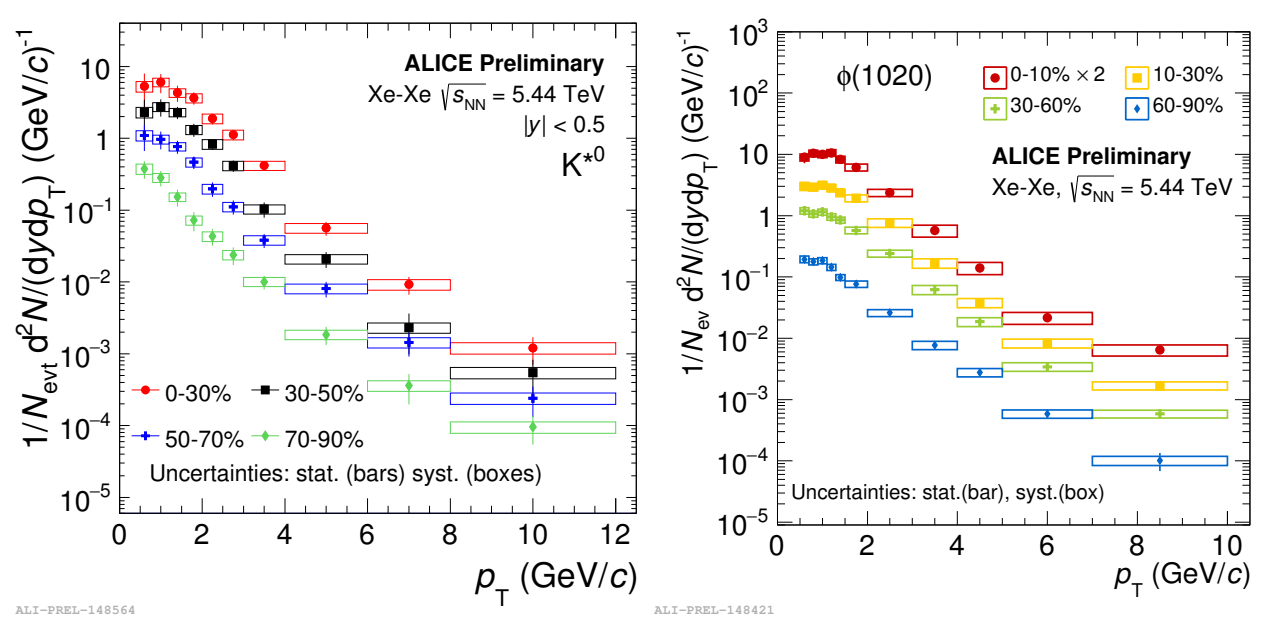

Figure 1. (color online) Transverse momentum spectra for $\mathrm{K}^{* 0}$ (left) and $\phi$ (right) in different centrality classes of Xe-Xe collisions at $\sqrt{s_{N N}}=5.44 \mathrm{TeV}$

The spectra have been measured for different centrality up to $p_{\mathrm{T}}=10 \mathrm{GeV} / c$.

The mean transverse momenta of $\phi$ and stable hadrons in $\mathrm{Xe}-\mathrm{Xe}$ collisions as a function of the charged-particle multiplicity density are shown in Fig. 2 (left). Results for Xe-Xe
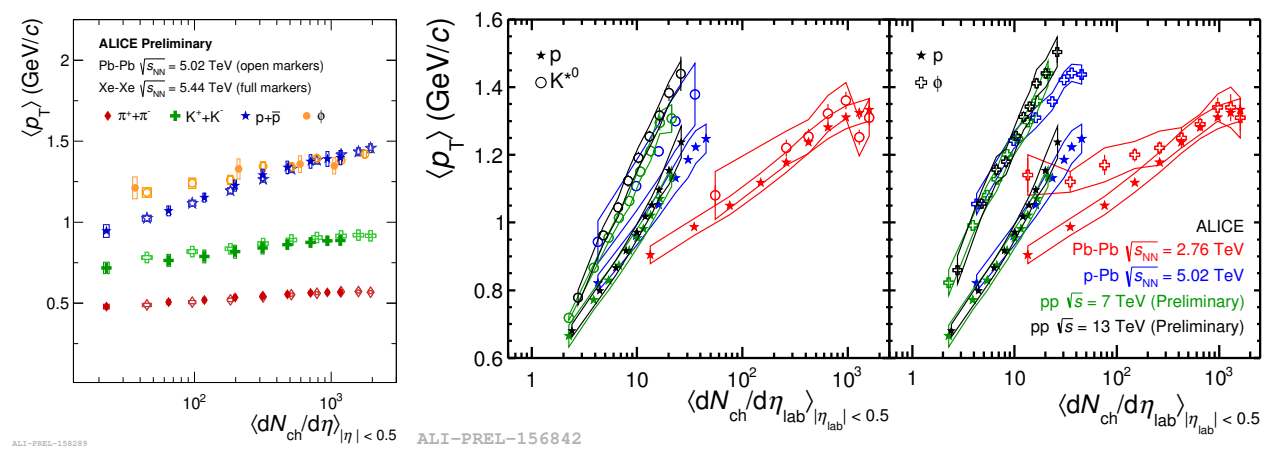

Figure 2. (color online) The mean transverse momentum as a function of the charged-particle multiplicity density. (left) pion, kaon, proton and $\phi$. (right) $\mathrm{K}^{* 0}$ (left panel) and $\phi$ (right panel) compared to $\mathrm{p}$ (same data in both panels)

collisions confirm the trends observed in $\mathrm{Pb}-\mathrm{Pb}$ collisions. In central collisions we observe mass ordering. The $\phi$ and $\mathrm{p}$, which have similar masses, are observed to have similar $\left\langle p_{\mathrm{T}}\right\rangle$ values, as expected if their spectral shape is dominated by radial flow.

Fig. 2 (right) presents the $\left\langle p_{\mathrm{T}}\right\rangle$ of $\mathrm{K}^{* 0}, \phi$ and $\mathrm{p}$ as a function of the charged-particle multiplicity density measured for pp collisions at $\sqrt{s}=13 \mathrm{TeV}$ and compared with the results obtained in pp, p- $\mathrm{Pb}[4]$ and $\mathrm{Pb}-\mathrm{Pb}[5]$ collisions at $\sqrt{s_{N N}}=7,5.02$ and $2.76 \mathrm{TeV}$, respectively. In pp collisions the $\left\langle p_{\mathrm{T}}\right\rangle$ increase with multiplicity increase at $\sqrt{s}=13 \mathrm{TeV}$ is similar 
to the one at $\sqrt{s}=7 \mathrm{TeV}$. The $\left\langle p_{\mathrm{T}}\right\rangle$ values follow a similar trend with multiplicity increase in $\mathrm{pp}$ and $\mathrm{p}-\mathrm{Pb}$ collisions, where they rise faster with multiplicity increase than in $\mathrm{Pb}-\mathrm{Pb}$ collisions. An analogous behavior has been observed in [10] for charged particles and can be understood as the effect of color reconnection between strings produced in multi-parton interactions. In central $\mathrm{Pb}-\mathrm{Pb}$ collisions, the $\left\langle p_{\mathrm{T}}\right\rangle$ values for these three particles are consistent within uncertainties, as it is expected in the presence of a common radial flow. However in $\mathrm{pp}$ and $\mathrm{p}-\mathrm{Pb}$ collisions the $\left\langle p_{\mathrm{T}}\right\rangle$ values for the $\mathrm{K}^{* 0}$ and $\phi$ resonances are higher than for $\mathrm{p}$. The mass ordering observed in central $\mathrm{Pb}-\mathrm{Pb}$ collisions, where particles with similar mass have similar $\left\langle p_{\mathrm{T}}\right\rangle$, is not observed in pp and $\mathrm{p}-\mathrm{Pb}$ collisions [4].

Fig. 3 shows the $\left\langle p_{\mathrm{T}}\right\rangle$ as a function of the charged-particle multiplicity density for $\rho^{0}$ [2] and $\Lambda^{*}$ [7] in $\mathrm{Pb}-\mathrm{Pb}$ collisions at $\sqrt{s_{N N}}=2.76 \mathrm{TeV}$. The results are in agreement with the
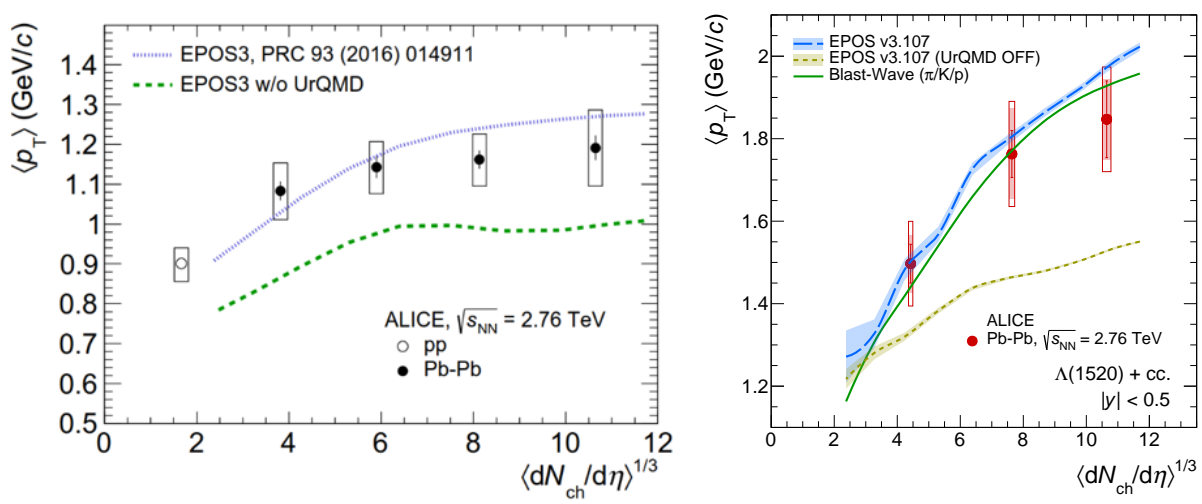

Figure 3. (color online) The mean transverse momentum of $\rho^{0}$ (left) [2] and $\Lambda^{*}$ (right) [7] as a function of the charged-particle multiplicity density. The measurements are also compared to model predictions: EPOS3 [11], Blast-Wave [12]

prediction from the EPOS3 generator with UrQMD [11], which includes modeling of rescattering and regeneration in the hadronic phase. The results for $\Lambda^{*}$ are also in agreement with the average momentum extracted from the Blast-Wave model [12] with parameters obtained from the simultaneous fit to pion, kaon, and (anti)proton $p_{T}$ distributions [13].

Fig. 4 presents $p_{T}$-integrated yields of $\mathrm{K}^{* 0}$ and $\phi$ in pp, p- $\mathrm{Pb}$ [4], $\mathrm{Xe}-\mathrm{Xe}$ and $\mathrm{Pb}-\mathrm{Pb}$ collisions as a function of the charged-particle multiplicity density. Yields are independent of collision system and appear to be driven by event multiplicity.

Fig. 5 (top left) shows the particle yield ratios $\mathrm{K}^{* 0} / \mathrm{K}$ and $\phi / \mathrm{K}$ in Xe-Xe collisions at $\sqrt{s_{\mathrm{NN}}}=5.44 \mathrm{TeV}$. Results for $\mathrm{p}-\mathrm{Pb}$ collisions at $\sqrt{s_{\mathrm{NN}}}=5.02 \mathrm{TeV}$ [4], $\mathrm{Pb}-\mathrm{Pb}$ collisions at $\sqrt{s_{\mathrm{NN}}}=2.76 \mathrm{TeV}$ [6], pp collisions at $\sqrt{s}=13 \mathrm{TeV}$ and $\mathrm{Pb}-\mathrm{Pb}$ collisions at $\sqrt{s_{\mathrm{NN}}}=5.02 \mathrm{TeV}$ are also shown. The $\mathrm{K}^{* 0} / \mathrm{K}$ ratio shows a significant suppression going from $\mathrm{p}-\mathrm{Pb}$ and peripheral $\mathrm{Xe}-\mathrm{Xe} / \mathrm{Pb}-\mathrm{Pb}$ collisions to the most central $\mathrm{Xe}-\mathrm{Xe} / \mathrm{Pb}-\mathrm{Pb}$ collisions. This suppression is consistent with rescattering of $\mathrm{K}^{* 0}$ daughters in the hadronic phase of central collisions as the dominant effect. There is a hint of decrease of the ratio with increasing multiplicity in $\mathrm{pp}$ and $\mathrm{p}-\mathrm{Pb}$ collisions. The values of the ratio are consistent for similar multiplicities in all collision systems (pp, p-Pb). The decrease of the ratio might be an indication of a hadron-gas phase with non-zero lifetime in high-multiplicity $\mathrm{pp}$ and $\mathrm{p}-\mathrm{Pb}$ collisions. The $\phi / \mathrm{K}$ ratio is nearly flat. This suggests that rescattering effects are not important for $\phi$, which has 10 times longer lifetime than $\mathrm{K}^{* 0}$ and decays mainly after the kinetic freeze-out. In $\mathrm{Pb}$ - $\mathrm{Pb}$ collisions at 

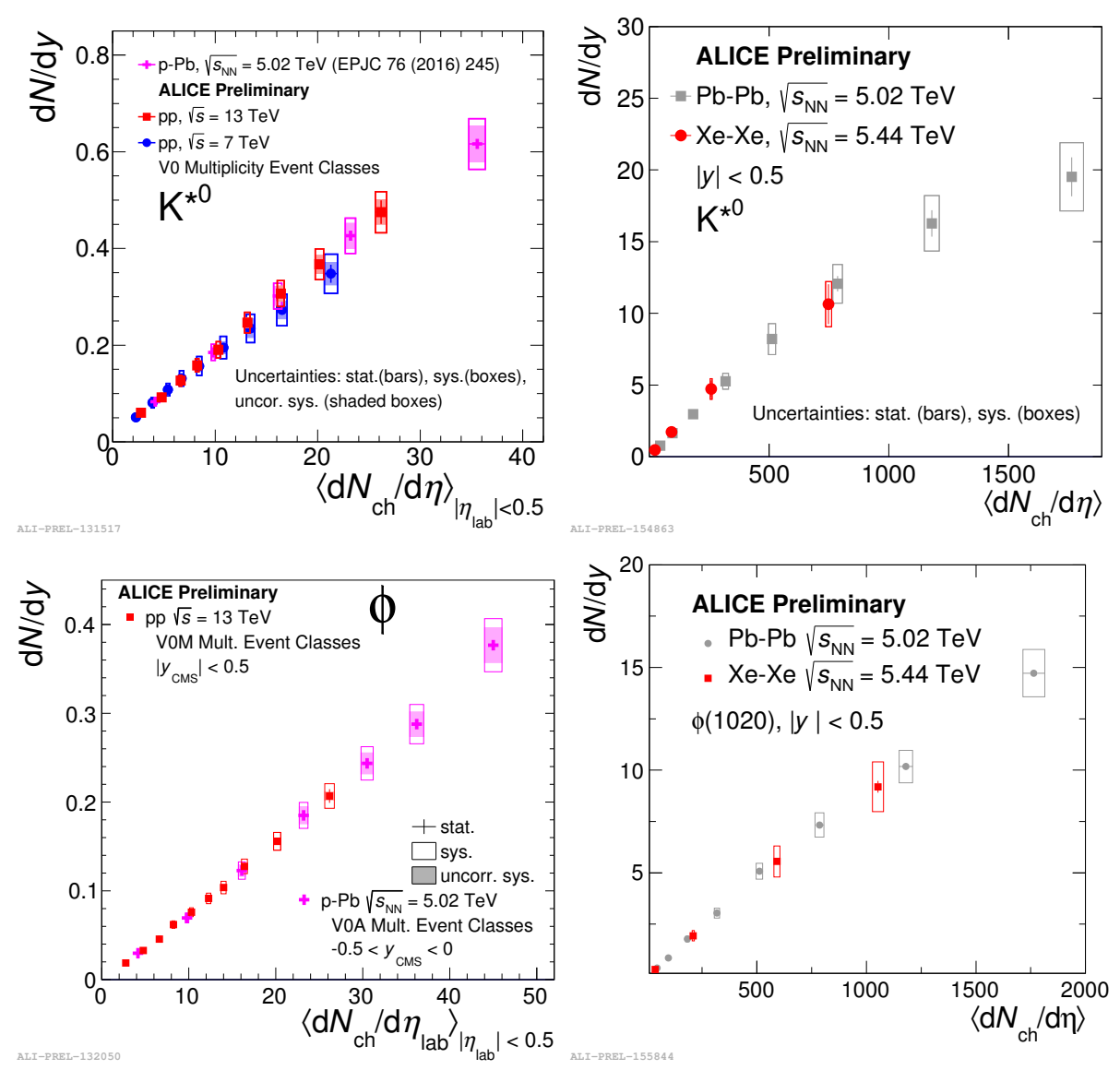

Figure 4. (color online) $p_{T}$-integrated yields (dN/dy) of $\mathrm{K}^{* 0}$ (top) and $\phi$ (bottom) as a function of the charged-particle multiplicity density in pp and $\mathrm{p}-\mathrm{Pb}$ [4] (left) and $\mathrm{Xe}-\mathrm{Xe}$ and $\mathrm{Pb}-\mathrm{Pb}$ (right) collisions

$\sqrt{s_{\mathrm{NN}}}=2.76 \mathrm{TeV}$ we observe $\rho^{0} / \pi[2]$ and $\Lambda^{*} / \Lambda$ [7] ratio suppression similar to the $\mathrm{K}^{* 0} / \mathrm{K}$ ratio, see Fig. 5 (top right) and Fig. 5 (bottom left), respectively. The $\Lambda^{*} / \Lambda$ suppression confirms the trend seen by STAR at $\sqrt{s_{\mathrm{NN}}}=200 \mathrm{GeV}$ [14]. For the $\Xi^{* 0} / \Xi$ ratio, Fig. 5 (bottom right), there is a hint of suppression, but systematics are to be reduced in peripheral $\mathrm{Pb}$ $\mathrm{Pb}$ collisions before making any conclusive statement. Although predictions of the EPOS3 model with UrQMD [11] overestimate the data, the trend of the suppression is qualitatively reproduced for $\mathrm{K}^{* 0}, \rho^{0}$, and $\Lambda^{*}$. Thermal model predictions [15-17] overestimate all particle ratios under study in central $\mathrm{Pb}-\mathrm{Pb}$ collisions, except the $\phi / \mathrm{K}$ ratio.

In central $\mathrm{Pb}-\mathrm{Pb}$ collisions the nuclear modification factor $R_{A A}$ for $\mathrm{K}^{* 0}[6], \phi[6]$ and $\rho^{0}$ [2] is consistent with light-flavored hadrons at $p_{\mathrm{T}}>8 \mathrm{GeV} / c$, demonstrating strong suppression. At low $p_{\mathrm{T}}<2 \mathrm{GeV} / c$, the $\mathrm{K}^{* 0}$ and $\rho^{0}$ are stronger suppressed than light-flavored hadrons which is consistent with the hypothesis that flow and rescattering effects are important. Fig. 6 (left) presents $R_{A A}$ of $\mathrm{K}^{* 0}$ for Pb-Pb collisions at $\sqrt{s_{\mathrm{NN}}}=2.76 \mathrm{TeV}[6]$ and $\sqrt{s_{\mathrm{NN}}}=5.02 \mathrm{TeV}$ in different centrality. We do not observe significant energy dependence of $R_{A A} . R_{A A}$ of $\mathrm{K}^{* 0}$ in $\mathrm{Xe}-\mathrm{Xe}$ and $\mathrm{Pb}-\mathrm{Pb}$ collisions are consistent within uncertainties if compared at the same multiplicity (and not just centrality percentile) as shown in Fig. 6 (right). 

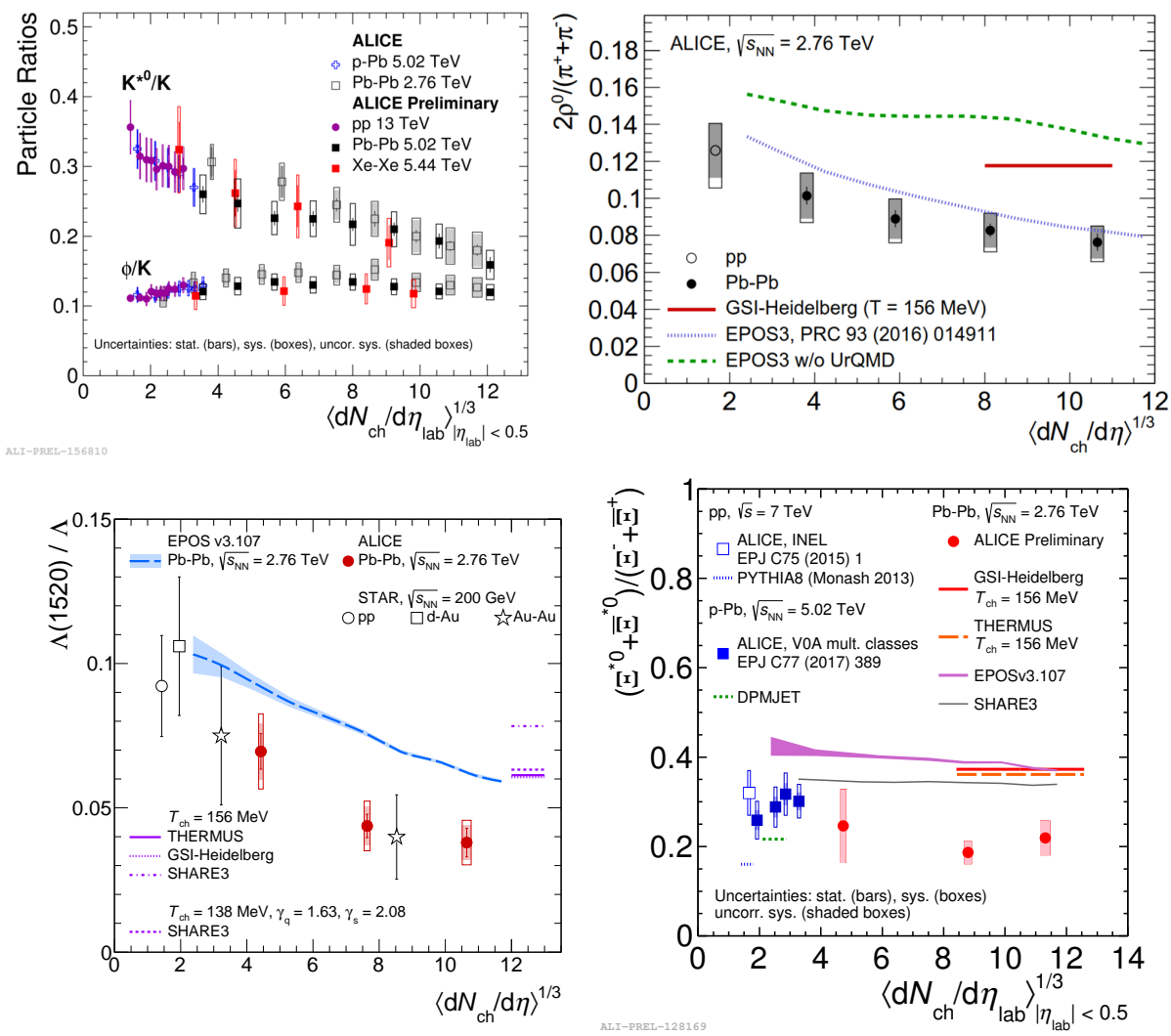

Figure 5. (color online) Particle yield ratios $K^{* 0} / K$ and $\phi / K$ (top left), $\rho^{0} / \pi$ (top right) [2], $\Lambda^{*} / \Lambda$ (bottom left) [7] and $\Xi^{* 0} / \Xi$ (bottom right) as a function of the charged-particle multiplicity density for various collision systems. STAR data from [14]. The measurements are also compared to model predictions: EPOS3 [11], THERMUS [15], GSI-Helderberg [16], SHARE [17]
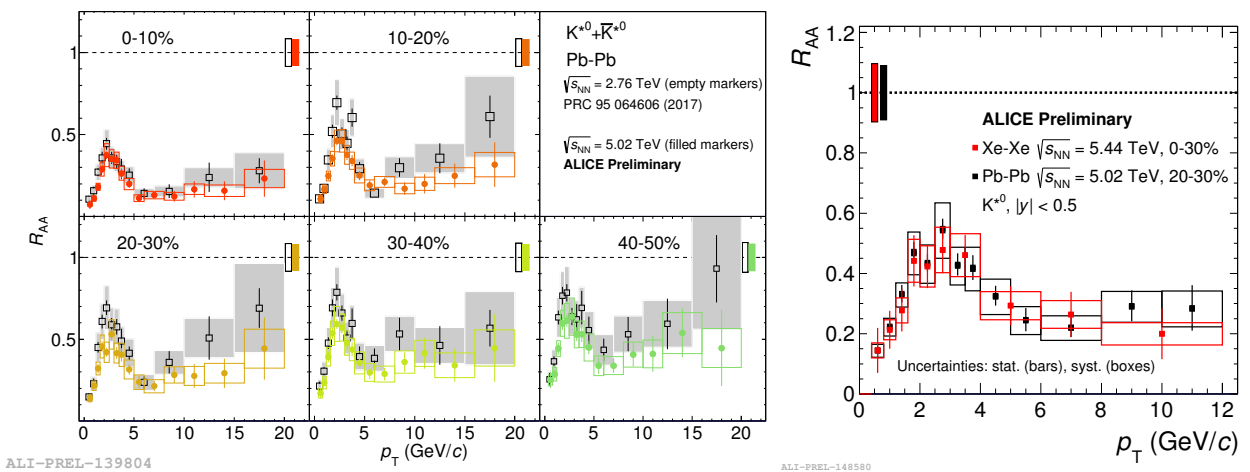

Figure 6. (color online) The nuclear modification factor $R_{A A}$ for $\mathrm{K}^{* 0}$ as a function of transverse momentum. (left) $\mathrm{Pb}-\mathrm{Pb}$ collisions at $\sqrt{s_{\mathrm{NN}}}=2.76 \mathrm{TeV}[6]$ and $\sqrt{s_{\mathrm{NN}}}=5.02 \mathrm{TeV}$ in different centrality. (right) $\mathrm{Xe}-\mathrm{Xe}$ and $\mathrm{Pb}-\mathrm{Pb}$ collisions at the same multiplicity (and not just centrality percentile) 
In summary, recent results on short-lived hadronic resonances obtained in the ALICE experiment in $\mathrm{pp}, \mathrm{p}-\mathrm{Pb}, \mathrm{Xe}-\mathrm{Xe}$ and $\mathrm{Pb}-\mathrm{Pb}$ collisions at the $\mathrm{LHC}$ energies have been presented. In $\mathrm{pp}$ and $\mathrm{p}-\mathrm{Pb}$ collisions the $\left\langle p_{\mathrm{T}}\right\rangle$ values for the $\mathrm{K}^{* 0}$ and $\phi$ resonances rise faster with multiplicity increase than in $\mathrm{Pb}-\mathrm{Pb}$ collisions. One possible explanation could be the effect of color reconnection between strings produced in multi-parton interactions. The mass ordering observed in central $\mathrm{Xe}-\mathrm{Xe}$ and $\mathrm{Pb}-\mathrm{Pb}$ collisions, where particles with similar masses $\left(\mathrm{K}^{* 0}\right.$, $\phi$ and $\mathrm{p}$ ) have similar $\left\langle p_{\mathrm{T}}\right\rangle$, is not observed in $\mathrm{pp}$ and $\mathrm{p}-\mathrm{Pb}$ collisions. Yields of $\mathrm{K}^{* 0}$ and $\phi$ in $\mathrm{pp}, \mathrm{p}-\mathrm{Pb}, \mathrm{Xe}-\mathrm{Xe}$ and $\mathrm{Pb}-\mathrm{Pb}$ collisions are independent of collision system and appear to be driven by event multiplicity. The $\mathrm{K}^{* 0} / \mathrm{K}, \rho^{0} / \pi$ and $\Lambda^{*} / \Lambda$ ratios exhibit a significant suppression going from peripheral to central $\mathrm{Pb}-\mathrm{Pb}$ collisions, is consistent with rescattering of the decay products of the short-lived resonances in the hadronic phase. The $\phi / \mathrm{K}$ ratio is not suppressed due to the longer lifetime of the $\phi$. Results for the $\mathrm{K}^{* 0} / \mathrm{K}$ and $\phi / \mathrm{K}$ ratios in $\mathrm{Xe}$ $\mathrm{Xe}$ collisions confirm the trend observed in $\mathrm{Pb}-\mathrm{Pb}$ collisions. There is a hint of suppression for the $\Xi^{* 0} / \Xi$ ratio, but systematics are to be reduced in peripheral $\mathrm{Pb}-\mathrm{Pb}$ collisions before making any conclusive statement. Although predictions of the EPOS3 model with UrQMD overestimate the data, the trend of the suppression is qualitatively reproduced. Thermal model predictions overestimate all particle ratios under study in central $\mathrm{Pb}-\mathrm{Pb}$ collisions, except the $\phi / \mathrm{K}$ ratio. In central $\mathrm{Pb}-\mathrm{Pb}$ collisions the nuclear modification factor $R_{A A}$ for $\mathrm{K}^{* 0}, \phi$ and $\rho^{0}$ consistent with light-flavored hadrons at $p_{\mathrm{T}}>8 \mathrm{GeV} / c$. At low $p_{\mathrm{T}}<2 \mathrm{GeV} / c$, the $\mathrm{K}^{* 0}$ and $\rho^{0}$ are stronger suppressed than light-flavored hadrons which would be consistent with the hypothesis that flow and rescattering effects are important. We do not observe significant energy dependence of $R_{A A}$ in $\mathrm{Pb}-\mathrm{Pb}$ collisions. $R_{A A}$ of $\mathrm{K}^{* 0}$ in $\mathrm{Xe}-\mathrm{Xe}$ and $\mathrm{Pb}-\mathrm{Pb}$ collisions are consistent within uncertainties once compared at the same multiplicity.

\section{References}

[1] G. Torrieri and J. Rafelski, Phys. Lett. B 509, 239 (2001)

[2] S. Acharya et al. (ALICE Collaboration), arXiv:1805.04365 [nucl-ex]

[3] B. Abelev et al. (ALICE Collaboration), Eur. Phys. J. C 72, 2183 (2012)

[4] J. Adam et al. (ALICE Collaboration), Eur. Phys. J. C 76, 245 (2016)

[5] B. Abelev et al. (ALICE Collaboration), Phys. Rev. C 91, 024609 (2015)

[6] J. Adam et al. (ALICE Collaboration), Phys. Rev. C 95, 064606 (2017)

[7] S. Acharya et al. (ALICE Collaboration), arXiv:1805.04361 [nucl-ex]

[8] B. Abelev et al. (ALICE Collaboration), Eur. Phys. J. C 75, 1 (2015)

[9] D. Adamova et al. (ALICE Collaboration), Eur. Phys. J. C 77, 389 (2017)

[10] B. Abelev et al. (ALICE Collaboration), Phys. Lett. B 727, 371 (2013)

[11] A.G. Knospe et al., Phys. Rev. C 93, 014911 (2016)

[12] E. Schnedermann et al., Phys. Rev. C 48, 2462 (1993)

[13] B. Abelev et al. (ALICE Collaboration), Phys. Rev. C 88, 044910 (2013)

[14] B. Abelev et al. (STAR Collaboration), Phys. Rev. C 78, 044906 (2008)

[15] S. Wheaton et al., Comput. Phys. Commun. 180, 84 (2009)

[16] J. Stachel et al., J. Phys.: Conf. Ser. 509, 012019 (2014)

[17] M. Petran et al., Comput. Phys. Commun. 185, 2056 (2014) 